Egyptian

Orthodontic Journal

\title{
DEBONDING CHARACTERISTICS OF SELF-ADHESIVE RESIN CEMENTS USED FOR ORTHODONTIC BONDING RELATIVE TO CURING INITIATION TIME
}

\author{
Abbas R. Zaher ${ }^{1}$; Nadia M. Al-Harony²; Mohamed I. El-Gayar ${ }^{3}$ \\ ABSTRACT:
}

Objective: The purpose of this study was to test whether the changing of the curing time initiation for two self-adhesive resin cements, Breeze (Pentron Clinical Technologies, Wallingford, USA) and Smartcem2 (Dentsply Caulk, International Milford, USA) can be used successfully to 6ond orthodontic brackets. Methods: Sixty extracted premolars were used in this study. The teeth were divided into 4 groups according to the cement used and its mode of curing. Breeze and Smartcem2were cured immediately and after 60 seconds after bracket placement. The brackets were tested in shear using a universal testing machine then the enamel surface was examined under 40x magnification to determine the amount of adhesive remnant. Six premolars from each group were then sectioned buccolingually into two halves. One half was chosen and gold coated to be examined under scanning electron microscope. Results: Analysis of variance for shear bond strength showed no significant differences among all the groups where the SBS ranged from 4.29 to $5.30 \mathrm{MPa}$. It was found that in 6oth types of self adhesive resin cements most of the failure occurred at the bracket tooth interface. Scanning electron microscope showed deeper resin penetration for Breeze and Smartcem 2 cured after 60 seconds with

1- Professor, Department of Orthodontics, Faculty of Dentistry, Alexandria University.

2- Professor, Department of Orthodontics, Faculty of Dentistry, Alexandria University.

3- Postgraduate Student Department of Orthodontics, Faculty of Dentistry, Alexandria University. 
Egyptian

Orthodontic Journal

a mean resin depth ranged from $3.25 \mu \mathrm{m}$ to $4.51 \mu \mathrm{m}$ respectively. $\mathcal{N}$ o resin tags were observed in groups immediately cured. Conclusions: The SBS values of brackets cemented with self-adhesive resin cements were below the optimum levels for use in orthodontic bonding and the formation of short resin tags with delayed curing did not increase the shear bond strength of self-adhesive resin cements.

\section{INTRODUCTION}

Since the introduction of the phosphoric acid etch technique for orthodontic bonding by Newman ${ }^{1}$, many different bonding systems and techniques have evolved including the chemically cured adhesive systems, the visible light-cured adhesive systems and the dual cure adhesives.

Many authors reported that etching with phosphoric acid produces considerable loss of enamel. ${ }^{2-5}$ Loss of enamel surface after phosphoric acid etching has been estimated between 10 and $30 \mu \mathrm{m} .{ }^{6}$ Thus the fluoride rich enamel surface is being removed, making the enamel more susceptible to deminarlization. ${ }^{7}$ Moreover, removal of the adhesive remnants after debonding will lead to more enamel loss which was estimated to be about $7.5 \mu \mathrm{m}$ surface loss when a tungsten-carbide bur was used. ${ }^{8}$ Also the remaining resin tags can cause the retention of plaque, formation of caries, and alteration of the enamel color which may be derived from the penetration of the resin tags into the enamel at depths that might reach to $50 \mu \mathrm{m} .{ }^{9,10,11}$ Potential enamel fractures during the debonding procedure have been reported as one of the shortcomings for the phosphoric acid etch technique. ${ }^{12}$

Several adhesive systems were introduced for orthodontic bracket bonding which do not require etching with phosphoric acid, including the use of resin modified glass ionomer and self-etch primers.

Recently one component self-etching and self-adhesive resin systems have been introduced in dentistry. These are dual cure materials as the polymerization can be photo and/or chemically activated and they are 
Egyptian

Orthodontic Journal

supplied as base and catalyst components in one automix double-push syringe with a mixing tip. These products combine etchant, primer, and adhesive resin in a single paste. Self adhesives can save the clinicians chair time by reducing the number of steps during the bonding procedures and therefore decreasing the possibility for contamination. Also after the debonding procedures, it was found that this system left the least amount of adhesive on the tooth surface compared to the conventional system or the two step self- etch primer/adhesive system. However, they offered lower shear bond strength (SBS) compared to the conventional and the self etch primer systems. ${ }^{12,13}$ ' No enamel fractures or cracks have been reported after the debonding procedures when the one step self-adhesive system was used for bonding of orthodontic brackets. ${ }^{12,13}$ Hence it constitutes a promising material that does not require etching and at the same time requires less steps for bonding. The null hypothesis is that delaying the application of the curing light to self-adhesive resin cements will not increase the resin tags penetration and therefore will not increase its shear bond strength.

\section{MATERIALS \& METHODS}

\section{Teeth}

A total of 60 human upper premolars free from cracks, caries and extracted for the purpose of orthodontic treatment were used in this study. The teeth were washed to remove any traces of blood and stored in distilled water, which was changed periodically to avoid deterioration. In no case was a tooth stored for more than a month after extraction.

\section{Brackets}

Sixty metal upper premolar brackets were used (Gemini, 3M Unitek Dental Products, Monrovia, Calif). The base surface area of each bracket was $10.61 \mathrm{~mm}^{2}$, as given by the manufacturer. 
Egyptian

Orthodontic Journal

\section{Bonding Procedure}

The teeth were divided into four groups, 15 for each, according to the material used and to the time at which the curing was initiated. The buccal surfaces were polished with a rubber cup and polishing paste. Ten seconds were given for bracket placement and excess removal. In each group the brackets were light cured 10 seconds from the mesial aspect and 10 seconds from the distal one.

Group BI: The brackets were cemented using Breeze self-adhesive resin cement and were light-cured immediately after proper bracket positioning and excess resin removal.

Group B60: The brackets were cemented using Breeze self-adhesive resin cement and were light-cured after 60 seconds after excess resin removal.

Group SI: The Brackets were cemented using Smartcem2 self-adhesive and light cured immediately after excess resin removal.

Group S60: The brackets were cemented using Smartcem 2 self -adhesive and were light cured after 60 seconds after excess resin removal.

\section{Mounting}

The teeth were mounted in acrylic blocks. A split copper ring was placed inside a copper mold and fixed firmly with internal hex screws, then a bracket positioning jig was screwed in the mold. The acrylic resin was poured into the copper ring up to the level of the cementoenamel junction (Figure 1). The bracket positioning jig was used to align the facial surface of the tooth perpendicular with the bottom of the mold. Each bracket was held and fixed to the jig with an O-tie. The jig ensures that the debonding force will be parallel to the tooth surface in an occlusal-apical direction. After complete setting of the acrylic resin, the O-tie was removed and the jig was unscrewed from the mold and the tooth within its acrylic block was removed from the split copper ring. The specimens were immersed in distilled water for 24 hours for complete curing at $37^{\circ} \mathrm{C}$ to prevent their dehydration until they were tested in shear. 
Egyptian

Orthodontic Journal

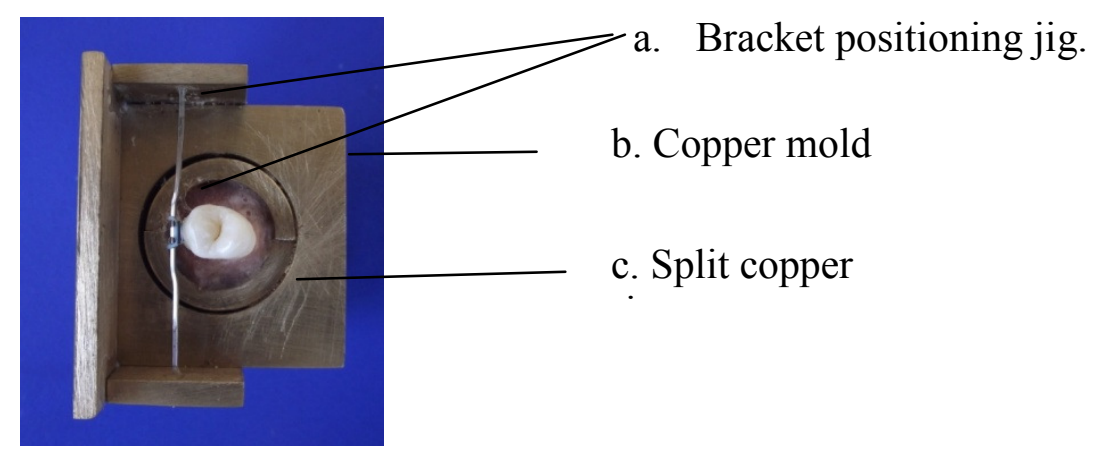

Figure (1): Occlusal view showing the bracket holded and fixed to the bracket positioning jig.

\section{Shear Bond Strength Test}

SBS was measured with a universal testing machine (Autograph AGS-1KND, Shimadzu, Kyoto, Japan) with a one-KN load cell connected to a metal rod with the end angled at $30^{\circ}$. The crosshead speed was one $\mathrm{mm} / \mathrm{min}$. The teeth were set at the base of the machine so that the sharp end of the rod incised in the junction of the bracket-tooth interface. The force required to debond each bracket was registered in newtons and converted into megapascals as a ratio of newtons to surface area of the bracket $(\mathrm{MPa}=\mathrm{N} / \mathrm{mm} 2)$.

\section{Adhesive Remnant Index}

Premolars from each group were examined under stereomicroscope at $40 \mathrm{x}$ magnification to evaluate the adhesive remnants and enamel fractures after bond failure. Photographs were taken and the area of the adhesive remnants was calculated for each tooth using micro image processing (MIP) software (Soft Imaging System sis, analysis, Gmbh, Germany). Afterward, each tooth was assessed using modified Adhesive Remnant Index (ARI) ${ }^{13}$ value according to the following criteria:

- Score (1): all adhesive left on the tooth.

- Score (2): more than $90 \%$ of the adhesive remained.

- Score (3): more than $10 \%$ but less than $90 \%$ of the adhesive left on the tooth. 
Egyptian

Orthodontic Journal

- Score (4): less than $10 \%$ of the adhesive left on the tooth.

- Score (5): no adhesive left on the tooth.

Possible enamel fractures were also observed with the stereomicroscope.

\section{Resin Tags Depth Measurement}

Six premolars from each group were sectioned buccolingually into two halves parallel to the long axis of the tooth using a diamond disc using low speed straight hand piece with appropriate cooling system. The mesial half of each tooth was selected and polished with 1200 grit silicon carbide papers (Superflex 273d, Horico, Berlin, Germany) under running water. After the segments were washed with water, $1 \%$ nitric acid solution was applied for 30 seconds, the specimens were rinsed with water and air dried overnight to remove the smear layer, then they were gold sputter-coated and examined under the scanning electron microscope (SEM). The enamel surface was scanned at 1500x magnification and $20 \mathrm{kv}$. Representative photographs were taken and the resin tags were measured using Micro Image Processing Software.

\section{Statistical analysis}

To compare the SBS between the 4 groups BI, B60, SI, S60, Kolmogorov-Smirnov and Shapiro-Wilk normality tests were applied to the bond strength data. Because the data did not show a normal distribution, a significant difference was evaluated using 2-way analysis of variance (ANOVA). The chi-square test was used to compare the Adhesive Remnant Index scores (ARI scores). ANOVA was used to compare the resin tags depth in enamel. Since there was significant difference in the data of resin tags, Post Hoc comparisons were done. Significance for all statistical tests was predetermined at $P \leq .05$.

\section{RESULTS}

\section{Shear Bond Strength}

The results of the 2-way ANOVA test showed no statistical significant difference among the 4 groups (Table 1). In BI the mean shear 
bond strength was $4.36 \pm 1.45 \mathrm{MPa}$ with a maximum 7.79 and a minimum 2.28 MPa. In B60 the mean shear bond strength was $5.30 \pm$ 1.44 MPa ranging from 7.91 and $2.35 \mathrm{MPa}$. In SI the mean shear bond strength was $4.15 \pm 1.95 \mathrm{MPa}$ ranging from 8.821 and $1.35 \mathrm{MPa}$. While in group S60 it showed a mean shear bond strength of $4.29 \pm 1.57 \mathrm{MPa}$ ranging from $6.98 \mathrm{MPa}$ and $1.366 \mathrm{MPa}$.

Table 1: Comparison of the mean SBS in MPa, Standard Deviation and Range for the four groups.

\begin{tabular}{|l|l|l|l|l|c|}
\hline \multicolumn{2}{|c|}{ Group } & Mean & $\begin{array}{l}\text { Standard } \\
\text { Deviation }\end{array}$ & Range & \multirow{2}{*}{ P-value } \\
\hline \multirow{2}{*}{ Breeze } & Group BI & 4.36 & 1.45 & $7.79-2.28$ & \multirow{2}{*}{$P=.204$} \\
\cline { 2 - 5 } & Group B60 & 5.30 & 1.44 & $7.91-2.35$ & \\
\hline \multirow{2}{*}{ Smartcem2 } & Group SI & 4.15 & 1.95 & $8.82-1.35$ & \\
\cline { 2 - 5 } & Group S60 & 4.29 & 1.57 & $6.98-1.36$ & \\
\hline
\end{tabular}

\section{Adhesive Remnant Index}

The Adhesive Remnant Indices of the four groups examined under stereomicroscope and the Chi-square comparisons of the ARI scores between the four groups showed that they had significantly different ARI scores (Table 2). For the Breeze resin cement cured immediately (BI group), no adhesive remained on the tooth after debonding with all the teeth in this group having ARI score 5, indicating failure at the enamel-adhesive interface. For Breeze cured after 60 seconds (B60 group), 11 teeth had ARI score 4 suggesting that most of the failure occurred at the enamel-adhesive interface. Four teeth in group B60 showed score 3. For Smartcem2 cured immediately (SI group), 14 teeth had ARI score 5 indicating failure at the enamel-adhesive interface. One tooth in this group had ARI score 4 suggesting that most of the failure was of the adhesive type.

For Smartcem 2 cured after 60 seconds (S60 group), 7 teeth had ARI score 5, four teeth had ARI score 4 and four teeth had ARI score 3. 
Table 2: Modified Adhesive Remnant Index scores and Chi-square comparisons for the four groups.

\begin{tabular}{|c|c|c|c|c|c|c|c|}
\hline & \multirow{2}{*}{$\mathbf{n}$} & \multicolumn{5}{|c|}{ Modified ARI scores $^{\mathrm{a}}$} & \multirow{2}{*}{$\begin{array}{c}\mathrm{x}^{2} \text { and } \\
P \text { - value }\end{array}$} \\
\hline & & 1 & 2 & 3 & 4 & 5 & \\
\hline Group BI & 15 & - & - & - & - & 15 & \multirow{4}{*}{$\begin{array}{c}\mathrm{x}^{2}=27.26 \\
P=.007\end{array}$} \\
\hline Group B60 & 15 & - & - & 4 & 11 & - & \\
\hline Group SI & 15 & - & - & - & 1 & 14 & \\
\hline Group S60 & 15 & - & - & 4 & 4 & 7 & \\
\hline
\end{tabular}

${ }^{a} 1$, all composite remained on the tooth; 2 , more than $90 \%$ of the composite remained on the tooth; $3,10-90 \%$ of the composite remained on the tooth; 4 , less than $10 \%$ of the composite remained on the tooth; 5 , no composite remained on the tooth.

There was a significant difference for the Chi-square comparisons of the ARI scores between BI and B60 with a $P$-value $=.0001$ (Table 3) and between SI and S60 with a $P$-value $=.017$. Hence leaving the self-adhesive resin cements for 60 seconds before light curing allowed for more adhesive remained on debonding (Table 4) $(P$-value $=.006)$.

Table 3: Modified Adhesive Remnant Index scores and Chi-square comparisons of BI and B60 groups.

\begin{tabular}{|c|c|c|c|c|c|c|c|}
\hline & \multirow[b]{2}{*}{ n } & \multicolumn{5}{|c|}{ Modified ARI scores } & \multirow{2}{*}{$\begin{array}{c}\mathrm{x}^{2} \text { and } \\
P \text { - value }\end{array}$} \\
\hline & & 1 & 2 & 3 & 4 & 5 & \\
\hline Group BI & 15 & - & - & - & - & 15 & \multirow{2}{*}{$\begin{aligned} \mathrm{x}^{2} & =30.000 \\
P & =.0001 *\end{aligned}$} \\
\hline Group B60 & 15 & - & - & 11 & 4 & - & \\
\hline
\end{tabular}

*Statistically significant at $\mathrm{P}$ value $<0.05$. 
Table 4: Modified Adhesive Remnant Index scores and Chi-square comparisons for SI and S60 groups.

\begin{tabular}{|l|c|c|c|c|c|c|c|}
\hline & & \multicolumn{5}{|c|}{ Modified ARI scores } & \multirow{2}{*}{$\mathbf{x}^{2}$ and } \\
\cline { 2 - 7 } & $\mathbf{n}$ & $\mathbf{1}$ & $\mathbf{2}$ & $\mathbf{3}$ & $\mathbf{4}$ & $\mathbf{5}$ & $\boldsymbol{P}$ - value \\
\hline Group SI & 15 & - & - & - & 1 & 14 & $\mathrm{x}^{2}=8.133$ \\
\cline { 1 - 7 } Group S60 & 15 & - & - & 4 & 4 & 7 & $P=.017$ \\
\hline
\end{tabular}

\section{Scanning Electron Microscope Examination}

The results of the 2-way ANOVA for the depth of resin tags showed a significant difference among the 4 groups (Table 5). BI and SI groups did not show any resin tags penetrating in the enamel surface (Figure 2a \& 2c). However, there was a variation in the depth of resin penetration into the enamel among B60 group which showed a mean resin penetration of $3.2586 \pm 0.99812 \mu \mathrm{m}$ (Figure 2b) and among S60 group which showed a mean resin penetration of $4.5180 \pm 1.73828 \mu \mathrm{m}$ (Figure 2d). The results of the Post Hoc test are presented in Table 6 and showing a significant difference among all the groups except between BI and SI groups which showed no difference.

Table 5: Comparison between mean resin tags depth, Standard Deviation and Range for the 4 groups.

\begin{tabular}{|l|c|c|c|c|c|}
\hline & n & Mean & $\begin{array}{c}\text { Standard } \\
\text { Deviation }\end{array}$ & Range & $p$-value \\
\hline Group BI & 0 & 0 & 0 & 0 & \\
\cline { 1 - 5 } Group B60 & 29 & 3.25 & 0.99 & $2.00-5.30$ & \multirow{2}{*}{$P=.0001^{*}$} \\
\hline Group SI & 0 & 0 & 0 & 0 & \\
\hline Group S60 & 30 & 4.51 & 1.73 & $1.92-7.60$ & \\
\hline
\end{tabular}

*Statistically significant at $\mathrm{P}$ value $<0.05$. 


\section{Egyptian \\ Orthodontic Journal}

Table 6: Results of the Post Hoc comparisons of mean resin tags depth for the 4 groups.

\begin{tabular}{|cl|c|c|c|}
\hline Group (I) & $\begin{array}{c}\text { Compared to } \\
\text { group (J) }\end{array}$ & $\begin{array}{c}\text { Mean } \\
\text { Difference (I-J) }\end{array}$ & Std. Error & Sig. \\
\hline 1. Group BI & 2 Group B60 & -3.24333 & .63597 & $.0001^{*}$ \\
& 3 Group SI & .00000 & .83268 & 1.000 \\
& 4 Group S60 & 4.518 & .64397 & $.0001^{*}$ \\
\hline 2. Group B60 & 3 Group SI & 3.24333 & .63597 & $.0001^{*}$ \\
& 4 Group S60 & -1.33391 & .34286 & .001 \\
\hline 3. Group SI & 4 Group S60 & -4.57724 & .63754 & $.0001^{*}$ \\
\hline
\end{tabular}

*Statistically significant at $\mathrm{P}$ value $<0.05$.

\section{Figure 2:}
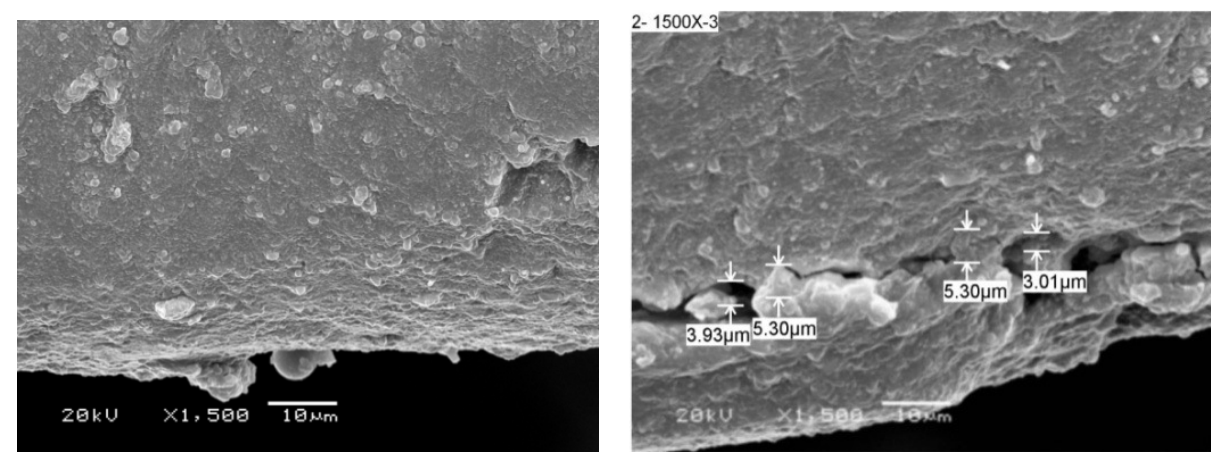

(a) SEM micrograph for Breeze cured immediately showing no resin penetration.

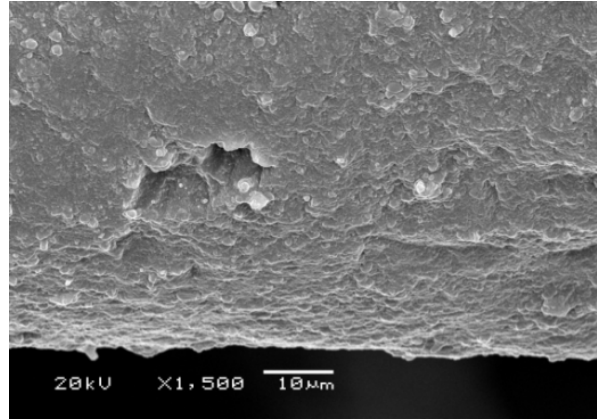

(c) SEM micrograph for Smartcem2 cured immediately showing no resin penetration showing no resin penetration (b) SEM micrograph for Breeze cured after 60 seconds showing a mean resin depth of $3.25 \mu \mathrm{m}$.

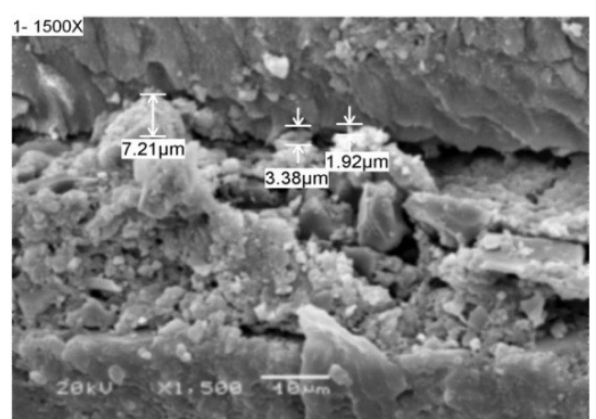

(d) SEM micrograph for Smartcem2 cured after 60 seconds showing a mean resin depth of $4.51 \mu \mathrm{m}$. 
Egyptian

Orthodontic Journal

\section{DISCUSSION}

With self-adhesives, the clinician can reduce chair time and eliminates the iatrogenic effect of the inorganic phosphoric acid etchant which produces enamel loss. On the other hand, these advantages only have real value if the adhesive bond strengths are within the optimum values.

Some investigators ${ }^{14,15}$ have suggested that a minimum SBS of 8.0 MPa can be adequate for clinical use in orthodontics. The maximum bond strength should be less than the breaking strength of enamel, which is about $14 \mathrm{MPa}^{16,17}$ The results of this research showed that self-adhesive resin cements produce a bond strength that was less than the optimum value for clinical use in orthodontics.

Although leaving the self-adhesive resin cements for 60 seconds before application of the curing light allowed for some penetration of the resin, it did not increase the SBS. This may be attributed to the lower tensile strength of the self-adhesive resin cements because of their lower filler content ( $\geq 65 \%$ by weight for Breeze and $69 \%$ by weight for Smartcem2) as the hardness and fracture toughness tend to be linearly proportional to filler content. ${ }^{18-19}$

In this study, the results of SBS for self-adhesive resin cements were nearly half the values reported by Al-Saleh and El-Mowafy ${ }^{20}$ $(\mathrm{X}=8.4 \mathrm{MPa})$ as they tested Breeze which was cured and stored in distilled water for 7 days. The higher values obtained in Al-Saleh and El-Mowafy ${ }^{20}$ study may be attributed to the longer storage time (7 days) before debonding. This might have allowed for full polymerization of the cement compared to 24 hours storage time in the present study. Bishara et $\mathrm{al}^{13}$ concluded that a one-step self-adhesive system has the potential to be successfully used in bonding orthodontic brackets if its shear bond strength can be increased when they tested Maxcem self-adhesive resin cement in orthodontic bonding and reported a mean SBS value of 3.1 $\mathrm{MPa}$. However, Vicente et $\mathrm{al}^{12}$ concluded that a self-adhesive resin cement can produce a SBS sufficient for clinical use in orthodontic bonding. In Vicente et $\mathrm{al}^{12}$ study RelyX Unicem self-adhesive resin cement was evaluated and a mean SBS value of $8.16 \mathrm{MPa}$ was obtained. 
Egyptian

Orthodontic Journal

The higher SBS value of the self-adhesive resin cement reported in that study may be attributed to the way of debonding the brackets in which the debonding force was exerted in the area between the base and the wings of the bracket; hence the debonding force had a torsion component (i.e. not a pure shearing force), however in the present study the debonding force was applied at the bracket tooth interface to create a pure shearing force. Bishara et $\mathrm{al}^{21}$ also evaluated the use of RelyX Unicem and found a mean SBS value of 3.7 MPa when brackets were debonded within 30 minutes. The early application of force for debonding in this study compared with that of the present study might have been responsible for the lower SBS obtained for the self-adhesive resin cement. This is consistent with previous studies ${ }^{20,21}$ demonstrating that composite adhesives have significantly lower SBS at 30 minutes as compared with 24 hours after bonding. In addition, the use of higher crosshead speed than that used in the present study $(1 \mathrm{~mm} / \mathrm{min})$ might also have resulted in lower SBS value.

Higher SBS for self-adhesive resin cements were found by Faltermeier et $\mathrm{al}^{22}$ when they tested Maxcem and RelyX Unicem self-adhesive resin cements and reported a mean SBS value of $7.06 \mathrm{MPa}$ and 7.12 MPa respectively. In that study ${ }^{22}$ the higher SBS value of the self-adhesive resin cements may be due to the increased curing time, in which the samples were light cured for 60 seconds using high intensity LED curing device $\left(1600 \mathrm{~mW} / \mathrm{mm}^{2}\right)$ allowing for more polymerization of the resin with a resultant increase in the mean SBS.

Many authors ${ }^{12,20,21}$ reported that for self-adhesive cements most of the adhesive remained on the bracket indicating failure at the enamel-adhesive interface.

ARI scores of the self-adhesive resin cements cured immediately showed almost no adhesive left on enamel after debonding. This might be because immediate curing did not allow sufficient time for resin penetration into the enamel.

Breeze and Smartcem 2 cured after 60 seconds left more adhesive on the enamel than when they were cured immediately as more time was allowed for some penetration of the resin into the enamel. Greater 
adhesive remnant was reported for Breeze cured after 60 seconds than Smartcem 2 cured after 60 seconds which may be attributed to the presence of 4-methacryloyloxyethy trimellitate (4-MET) resin in the Breeze resin cement. Some authors have reported improved adhesion to enamel due to the presence of 4-MET. ${ }^{23}$ It was reported that 4-MET is able to establish an ionic bond with calcium in the hydroxyapatite crystals. $^{23}$

For Breeze and Smartcem2 applied and cured immediately, no resin tags were detected by the SEM. This may be attributed to its high viscosity and not enough time allowed for the acidic monomer to penetrate into the enamel. On the other hand, leaving them for 60 seconds before curing allowed longer time for resin penetration.

Breeze cured after 60 seconds showed shorter resin tags in the enamel than Smartcem 2 cured after 60 seconds. This may be attributed to the type of acidic monomer used for each adhesive. In Breeze the acidic monomer is 4-MET which possesses an initial $\mathrm{pH}$ of 2 to 2.2, while in Smartcem 2 the acidic monomer is phosphoric acid modified acrylate resin which has very low $\mathrm{pH}$ values of 0.7 to 1.2 and a lower molecular weight than 4 -MET. ${ }^{24}$ Hence has higher dissolving capacity to the hydroxyapetite and result in longer resin tags and more opportunity for the resin to penetrate into the enamel. ${ }^{24}$

\section{CONCLUSION}

1) SBS of Breeze and Smartcem2 self-adhesive resin cements was below the optimum level for clinical use in orthodontic bonding.

2) Delayed curing didn't enhance the bond strength of either Breeze or Smartcem 2 self-adhesive resin cements.

3) For Breeze and Smartcem 2 self-adhesive resin cements, bond failure was observed to occur at the enamel-adhesive interface specially with immediate curing as it almost left no adhesive remnants on the enamel surface on debonding. 
Egyptian

Orthodontic Journal

4) Leaving the self-adhesive resin cements for 60 seconds before light curing" gave the time for the formation of short resin tags. while with immediate curing, no resin tags were formed. However it did not enhance the bond strength.

\section{RECOMMENDATIONS}

1) For self-adhesive resin cements, the manufacturer might consider increasing its SBS in order to be used in bonding orthodontic.

2) For its use as an orthodontic adhesive, it is recommended that the manufacturer change the consistency of self-adhesive resin cements as they have thin film thickness which result in bracket sliding after proper positioning.

\section{REFERENCES}

1. Newman GV, Snyder WH, Wilson CE. Acrylic adhesives for bonding attachments to tooth surfaces. Angle Orthod 1968;38:12-18.

2. Reisner KR, Levitt HL, Mante F. Enamel preparation for orthodontic bonding: a comparison between the use of a sandblaster and current techniques. Am J Orthod Dentofacial Orthop 1997;111:366-373.

3. Van Waes H, Matter T, Krejci I. Three dimensional measurement of enamel loss caused by bonding and debonding of orthodontic brackets. Am J Orthod Dentofacial Orthop 1997;112:666-669.

4. Canay S, Kocadereli I, Akca E. The effect of enamel air abrasion on the retention of bonded metallic orthodontic brackets. Am J Orthod Dentofacial Orthop 2000;117:15-19.

5. Van Waveren WL, Feilzer AJ, Phrahl-Andersen B. The air abrasion technique versus the conventional acid-etching technique: a quantification of surface enamel loss and comparison of shearbond strength. Am J Orthod Dentofacial Orthop 2000;117:20.

6. Bishara SE, VonWald L, Laffon JF, Jacobsen JR. Effect of altering the type of enamel conditioner on the shear bond strength of a resin reinforced glass ionomer adhesive. Am J Orthod Dentofacial Orthop 2000;118:288-294. 
7. Legler LR, Retief DH, Bradley EL, Denys FR, Sadowsky PL. Effects of phosphoric acid concentration and etch duration on shear bond strength of an orthodontic bonding resin to enamel. Am J Orthod Dentofacial Orthop 1989;96:485-492.

8. Waes VH, Matter T, Krejci I. Three-dimensional measurement of enamel loss caused by bonding and debonding of orthodontic brackets. Am J Orthod Dentofacial Orthop 1997; 112: 666-9.

9. Abbas R Zaher, Essam M Abdalla, Maha A Abdel Motie, Noman Atiq Rehman, Hassan Kassem and Athanasios E Athanasiou. Enamel color changes after debonding using various bonding systems. J. Orthod 2012; 39:82-88.

10. Reisner KR, Levitt HL, Mante F. Enamel preparation for orthodontic bonding: a comparison between the use of a sandblaster and current techniques. Am J Orthod Dentofacial Orthop 1997; 111: 366-73.

11. Silverstone LM, Saxton CA, Dogon IL, Fejerskov O. Variation in the pattern of acid etching of human dental enamel examined by scanning electron microscopy. Caries Research 1975; 9: 373-5.

12. Vicente A, Bravo LA, Romero M, Ortiz AJ, Canteras M. A comparison of the shear bond strength of a resin cement and two orthodontic resin adhesive systems. Angle Orthod 2004;75:109-13.

13. Bishara SE, Ajlouni R, Laffoon JF, Warren JJ. Comparison of shear bond strength of two self-etch primer/adhesive systems. Angle Orthod 2006;76:123-6.

14. Leung Y, Morris MD. Characterization of the chemical interactions between 4-MET and enamel by Raman spectroscopy. Dent Mater J 1995;11(3):191-195.

15. Nakabayashi N, Pashley DH. Hybridization of dental hard tissues. Tokyo: Quintessence Publishing; 1998.

16. Powers JM, Messersmith ML. Enamel etching and bond strength. In: Brantley WA, Eliades T, eds. Orthodontic Materials:mScientific and Clinical Aspects. Stuttgart, Germany: Stuttgart-Thieme; 2001:105-122. 
Egyptian

Orthodontic Journal

17. Pickett KL, Sadowsky PL, Jacobson A, Lacafield W. Orthodontic in vivo bond strength: comparison with in vitro results. Angle Orthod 2001;71:141-148.

18. Arhun N, Arman A, Sesen C, Karabulut E, Korkmaz Y, Gokalp S. Shear bond strength of orthodontic brackets with 3 self-etch adhesives. Am J Orthod Dentofacial Orthop 2006; 129:547-50.

19. Yamaga T, Sato Y, Akagawa Y, Taira M, Wakasa K, Yamaki M..Hardness and fracture toughness of four commercial visible light-cured composite resin veneering materials. J Oral Rehabil 1995 Dec;22(12):857-63.

20. Al-Saleh M, El-Mowafy O. Bond strength of orthodontic brackets with new self-adhesive resin cements. Am J Orthod Dentofacial Orthop 2010;137:528-33.

21. Bishara SE, VonWald L, Olsen ME, Laffoon JF. Effect of time on the shear bond strength of glass ionomer and composite orthodontic adhesives. Am J Orthod Dentofacial Orthop1999;116:616-620.

22. Faltermeier A, Behr M, Müssig D. A comparative evaluation of bracket bonding with 1-, 2-, and 3-component adhesive systems. Am J Orthod Dentofacial Orthop 2007;132:144.e1-5.

23. O'Brien RD, Watts DC, Read MJF. Residual debris and bond strength-is there a relationship? Am J Orthod Dentofacial Orthop 1988;94:222-30.

24. Salz U, Mücke A, Zimmermann J, Tay FR, Pashley DH. pKa value and buffering capacity of acidic monomers commonly used in self-etching primers. J Adhes Dent 2006 Jun;8(3):143-50. 